\section{Heilmittelregresse werden ab 2017 deutlich schwieriger}

\begin{abstract}
- Neue Regeln für Praxisbesonderheiten im Heilmittel-Bereich: Anfang 2017 kommt eine neue, bundesweit gültige Vereinbarung. Dann wird eine neue Diagnoseliste in Kraft treten, als Anhang der auf Bundesebene geschlossenen Rahmenvorgaben für die Wirtschaftlichkeitsprüfung nach $₫ 106 \mathrm{~b}$ Abs. 2 SGB $\mathrm{V}$. Ab diesem Zeitpunkt werden auch alle Diagnosen, für die ein langfristiger Heilmittelbedarf anzunehmen ist, durch den Gemeinsamen Bundesausschuss (G-BA) beschlossen und als Anlage in die Heilmittel-Richtlinie integriert. Die bisherige Vereinbarung nach $₫ 84$ Abs. 8 Satz 3 SGB V wird damit abgelöst.

Es gab bisher schon einige wenige Diagnosen, die nur dann als Praxisbesonderheit anerkannt werden konnten, wenn sie mittels eines zweiten ICD-10Codes spezifiziert wurden - z.B. die
\end{abstract}

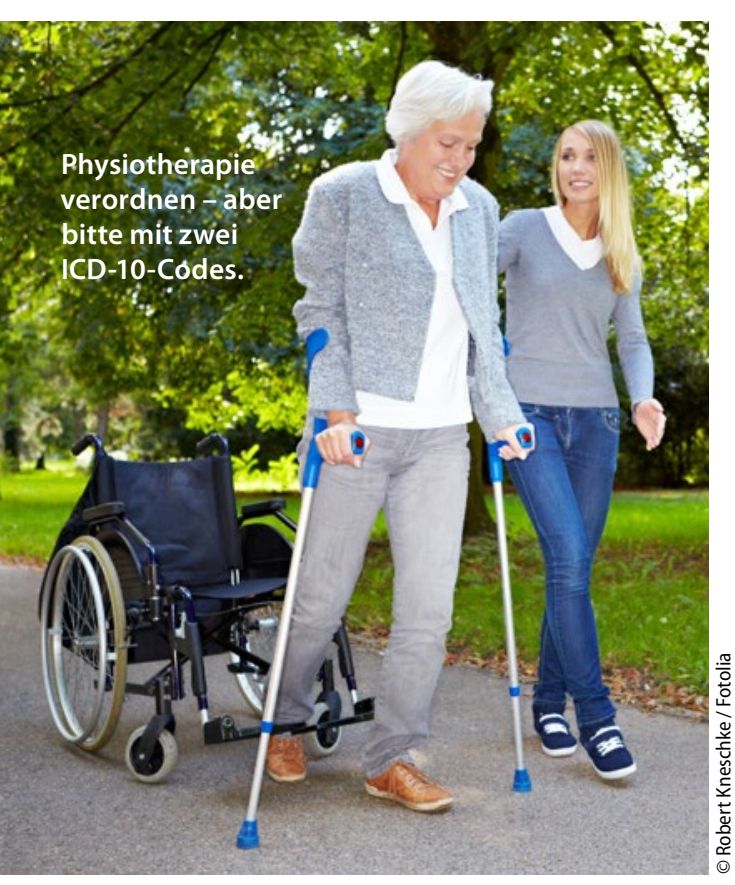

postoperative Versorgung einer chronischen Instabilität des Kniegelenkes. Diese Methode wird nun ausgebaut. Um zukünftig die Identifikation der besonderen Verordnungsbedarfe im Vorfeld einer Wirtschaftlichkeitsprüfung eindeutig sicherzustellen, wird es ein zweites elektronisch lesbares ICD-10-Feld auf den Verordnungsformularen geben. Es besteht für den Arzt keine Verpflichtung, dieses Feld zu auszufüllen - man muss es aber tun, wenn man bestimmte besondere Verordnungsbedarfe geltend machen möchte. Beispielsweise könnte man hier bei einer postoperativen Versorgung eine Myelopathie oder eine Radikulopathie bei Bandscheibenschäden angeben.

Die ergänzten Verordnungsformulare - es handelt sich um die Muster 13, 14 und 18 - werden gleichzeitig mit den geänderten Regelungen zu den besonderen Verordnungsbedarfen zum 1. Januar 2017 eingeführt.

\section{MMW-KOMMENTAR}

GKV-Spitzenverband und KBV haben vereinbart, die aktuell geltende Diagnoseliste auch 2017 grundsätzlich inhaltlich fortzuführen. Darüber hinaus werden einige weitere Diagnosen in den bundesweiten Katalog der besonderen Verordnungsbedarfe aufgenommen. Verordnungen aufgrund dieser Diagnosen entlasten den Arzt im Falle einer Wirtschaftlichkeitsprüfung, da sie in vollem Umfang berücksichtigt werden.

Unter der Kategorie "Geriatrische Syndrome" wurde eine Reihe von Diagnosen neu aufgenommen, die ab dem vollendeten 70 . Lebensjahr in Zusammenhang mit den entsprechenden Diagnosegruppen nach dem Heilmittelkatalog einen besonderen Verordnungsbedarf darstellen. Dabei handelt es sich u. a. um Demenz und Osteoporose mit

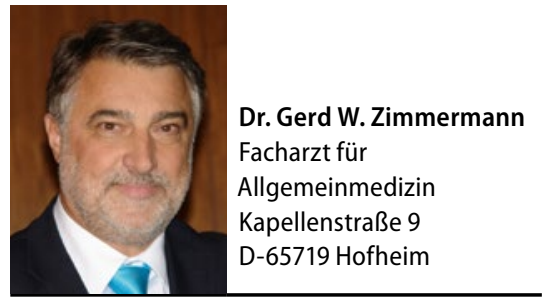

\section{Neue Diagnosen, bei denen ab 2017 ein Heilmittelregress ausgeschlossen ist}

- Demenz ab dem 70. Lebensjahr, Alzheimer-Demenz vor dem 65. Lebensjahr

- Osteoporose mit pathologischer Fraktur

- Entwicklungsstörungen bei Kindern

- Sekundäres Parkinson-Syndrom

- Chronische Atemwegserkrankungen mit Ursprung in der Perinatalperiode

- Versorgung von Schulterläsionen

- Systemkrankheiten des Bindegewebes

- Kyphosen, Skoliosen, juvenile Osteochondrosen

pathologischer Fraktur. Mit diesem Schritt soll einer erforderlichen Intensivierung der geriatrischen Heilmittelversorgung Rechnung getragen werden. Die Anerkennung als besonderer Verordnungsbedarf erfolgt unabhängig davon, ob der vertragsärztliche Verordnungsbedarf aufgrund von Behandlungsplänen nach § 118a SGB V (Geriatrische Institutsambulanzen) entstanden ist oder nicht.

Neben Demenzerkrankungen, die der oben genannten Altersbeschränkung unterliegen, wurde zusätzlich die Versorgung von Patienten mit Demenz bei Alzheimer-Krankheit mit frühem Beginn, hier definiert als vor dem 65. Lebensjahr, als besonderer Verordnungsbedarfaufgenommen.

Auch aus anderen Bereichen wurden spezifische Diagnosen als besondere Verordnungsbedarfe in die Liste aufgenommen (siehe Kasten). Ferner sollen einige Diagnosen, die bisher als Praxisbesonderheit galten, in die Diagnoseliste des langfristigen Heilmittelbedarfs übernommen werden. Hierzu läuft derzeit beim G-BA noch ein Stellungnahmeverfahren. 